\title{
DIGESTIBILIDADE DE DIETAS E METABOLISMO DE SUÍNOS ALIMENTADOS COM DIETAS CONTENDO EXTRATOS CÍTRICOS
}

\author{
DIGESTIBILITY OF DIETS AND METABOLISM OF PIGS FED WITH DIETS CONTAINING \\ CITRICEXTRACTS
}

\author{
Lanferdini, E. ${ }^{*}$; Andretta, I. ${ }^{\text {; }}$ Lehnen, C.R. ${ }^{1}$; Melchior, R. ${ }^{\text {; }}$ Silva, M.F.R. ${ }^{1}$ e Garcia, G.G. ${ }^{1}$ \\ 'Universidade Federal de Santa Maria. Departamento de Zootecnia. Santa Maria, RS. Brasil. \\ *eloizalanferdini@gmail.com
}

\section{PALAVRAS CHAVE ADICIONAIS}

Ácido ascórbico. Bioflavonóides. Colesterol. Digestão. Nutrição. Suinocultura.

\section{RESUMO}

O experimento foi realizado com o objetivo de avaliar a digestibilidade das dietas e o metabolismo de suínos em terminação alimentados com dietas contendo extratos cítricos. Foram utilizados 12 suínos, machos castrados, com peso médio inicial de 54 quilogramas. $O$ delineamento experimental foi inteiramente casualizado com dois tratamentos (dieta controle e dieta controle $+750 \mathrm{mg} \mathrm{kg}^{-1} \mathrm{de} \mathrm{um}$ composto de extratos cítricos, contendo ácido ascórbico e bioflavonóides) de seis repetições cada, sendo o animal a unidade experimental. O uso de extratos cítricos não alterou $(p>0,05) \circ$ balanço de nitrogênio, porém aumentou $(p=0,05)$ em $3,56 \%$ a energia retida e em $(p<0,05) 1,94 \%$ a energia digestível. Suínos alimentados com dietas contendo extratos cítricos apresentaram redução $(p<0,05)$ de $12,9 \%$ no nível de colesterol sanguíneo. A adição de extratos cítricos em dietas para suínos melhora a retenção de energia, o aproveitamento da energia digestível e reduz os níveis plasmáticos de colesterol.

\section{SUMMARY}

An experiment was carried out to evaluate the digestibility of diets, and metabolism of finishing pigs fed diets containing citric extracts. Twelve barrows with average initial weight of 54 kilograms were used. A completely randomized experimental design was used, with two treatments (control diet and control diet $+750 \mathrm{mg} \mathrm{kg}^{-1}$ of a compound of citric extracts, containing ascorbic acid and

\section{AdDitionAl KEYWORDS}

Ascorbic acid. Bioflavonoids. Cholesterol. Digestion. Nutrition. Swine production.

bioflavonoids) with six replications, and the animal as an experimental unit. The citric extracts did not alter ( $p>0.05)$ nitrogen balance, but increased $(p<0.05)$ in $3.56 \%$ the retained energy and in $1.94 \%$ the digestible energy. Pigs fed diets containing citrus extracts had decreased $(p<0.05)$ from $12.9 \%$ in the level of blood cholesterol. The addition of citric extracts in diets for pigs improves the retention of energy, the use of digestible energy and reduces the plasma cholesterol levels.

\section{INTRODUÇÃO}

A emergente exigência dos importadores e de grupos específicos de consumidores pela retirada de antimicrobianos de síntese das dietas de suínos tem promovido o interesse por aditivos alternativos que garantam a produtividade sem afetar a qualidade do produto final. Alguns aditivos utilizados são os probióticos, prebióticos, ácidos orgânicos, enzimas e extratos vegetais (Jeauround et al., 2002). Dentre estes, os extratos vegetais destacam-se por possuir propriedades antimicrobianas e imunomoduladoras, atuando sobre a microbiota intestinal e a digestão dos nutrientes (Mellor, 2000). Os efeitos dos extratos vegetais variam com sua origem e, portanto, com seus princípios ativos. Os 


\section{LANFERDINI, ANDRETTA, LEHNEN, MELCHIOR, SILVA E GARCIA}

principais componentes do extrato derivado de frutas cítricas são os compostos fenólicos (bioflavonóides) e o ácido ascórbico.

Os bioflavonóides são antioxidantes naturais, com ações anti-inflamatórias, antimicrobianas, antialergênicas e imunoestimulantes (Erlund, 2004; Cushnie e Lamb, 2005). Esses compostos, por apresentarem ação inibitória sobre algumas enzimas e propriedade quelante em metais, inibem as reações em cadeia induzidas por radicais livres (Erlund, 2004). Da mesma forma, o ácido ascórbico é um importante cofator enzimático que participa de diversos processos de óxido-redução, inativação de radicais livres e aumento da absorção de ferro (Padayatty et al., 2003). Essa substância participa também de diversos processos metabólicos, na formação do colágeno e síntese de epinefrina, corticoesteróides e ácidos biliares (Mahan et al., 2004).

As propriedades do extrato de frutas cítricas o torna um aditivo potencial para dietas de suínos. No entanto, poucos estudos abordam sua aplicação na alimentação animal, seus mecanismos de ação e a interação com o metabolismo de suínos. Portanto, o objetivo do trabalho foi avaliar a digestibilidade das dietas, o metabolismo e os níveis plasmáticos de colesterol e triglicerídeos de suínos alimentados com dietas contendo extratos cítricos.

\section{MATERIALEMÉTODOS}

O trabalho foi realizado no Setor de Suínos, do Departamento de Zootecnia, da Universidade Federal de Santa Maria. Foram utilizados 12 suínos machos castrados, geneticamente homogêneos, oriundos de cruzamento industrial, com peso médio inicial de $54 \mathrm{~kg}$ e 98 dias de idade. O delineamento experimental foi inteiramente casualizado, constituído de dois tratamentos (dieta controle e dieta controle $+750 \mathrm{mg} \cdot \mathrm{kg}^{-1}$ de um composto de extratos cítricos, contendo ácido ascórbico e bioflavonóides). Os extratos cítricos foram adicionados ao veículo, constituindo $50 \%$ do produto em ácido ascórbico e bioflavonóides. Cada tratamento teve seis repetições, sendo o animal a unidade experimental.

As dietas experimentais foram formuladas segundo as recomendações nutricionais do NRC (1998). O experimento de digestibilidade e metabolismo teve duração de 12 dias, sendo sete dias de adaptação dos animais às gaiolas e às dietas e cinco para coletas de fezes, urina e sobras de ração. As dietas foram fornecidas de acordo com o peso metabólico dos animais $\left(\mathrm{PV}^{0,60}\right)$, sendo ajustado diariamente com base no ganho médio diário estimado. Para este ajuste foi considerando um consumo de 2,6 vezes a mantença estimada em $250 \mathrm{kcal} \mathrm{EM} / \mathrm{kg} \mathrm{PV}^{0,6}$ (Noblet et al., 1993). As dietas experimentais foram distribuídas em três refeições diárias e os animais tiveram livre acesso à água. Foi utilizado o método de coleta total de fezes, coletadas duas vezes ao dia e conservadas $\mathrm{a}-10^{\circ} \mathrm{C}$ em congelador. Ao final do experimento, as fezes foram homogeneizadas e amostradas $(0,50 \mathrm{~kg})$, secas em estufa de ventilação forçada $\left(60^{\circ} \mathrm{C}\right.$ por 72 horas) e moídas para análises posteriores. A urina excretada foi drenada para baldes de plástico com $25 \mathrm{~mL}$ de $\mathrm{HCl}(6 \mathrm{~N})$. A cada 12 horas, o volume de urina era quantificado, homogeneizado e uma amostra de $5 \%$ retirada e conservada a $4{ }^{\circ} \mathrm{C}$ sob refrigeração. As análises químicas de fezes e urina foram realizadas segundo metodologia proposta pela AOAC (1990).

Após o estudo metabólico, os suínos foram alojados individualmente por 42 dias em baias convencionais. $\mathrm{O}$ fornecimento de ração foi à vontade e os animais tiveram livre acesso à água. Os animais foram abatidos com $102 \mathrm{~kg}$ em média. Durante a sangria foram coletadas amostras de sangue para análise de triglicerídeos e colesterol total. A análise foi realizada através de kits enzimáticos e as amostras foram processadas em analisador automático $\left(\mathrm{Cobas}^{\circledR}\right.$, Mira plus, 


\section{DIGESTIBILIDADE DE EXTRATOS CÍTRICOS E METABOLISMO DE SUÍNOS}

Roche). Os dados obtidos foram submetidos à análise de variância pelo procedimento General Linear Model utilizando o programa Minitab 15 (Minitab, 2007).

\section{RESULTADOSEDISCUSSÃO}

Não foram observadas alterações $(\mathrm{p}>0,05)$ nas variáveis analisadas para o balanço de nitrogênio, porém a adição de extratos cítricos aumentou ( $\mathrm{p}<0,05)$ em $3,56 \%$ a energia retida e em $1,94 \%$ a energia digestível de suínos em terminação (tabela I). O sistema digestório de suínos em crescimento e terminação já está desenvolvido e o efeito benéfico da utilização de extratos vegetais na dieta está geralmente relacionado à melhora na conversão alimentar. Além disso, a quercetina, rutina e naringenina são bioflavonóides presentes nas frutas cítricas que podem estimular o consumo de ração, pois estão relacionados com a melhora na palatabilidade e na digestão dos alimentos (Ader et al., 2000). A presença de extratos cítricos na dieta aumenta a produção de pepsina e ácido gástrico no organismo e contribui para a redução do $\mathrm{pH}$ estomacal e do intestino delgado, estimulando a secreção pancreática(Mellor, 2000). Estas substâncias podem ter colaborado com os processos digestivos, ativando alguns mecanismos de controle da ingestão e absorção energética.

A dieta contendo extratos cítricos reduziu $(\mathrm{p}<0,05)$ em $12,9 \%$ o nível sanguíneo de colesterol total em suínos (tabela I). $\mathrm{O}$ ácido ascórbico tem se destacado por alterar o metabolismo do colesterol, porém seu mecanismo de ação ainda não é completamente conhecido. Uma das hipóteses associa o ácido ascórbico com interferências nas reações bioquímicas que envolvem os sistemas indicadores com oxidases e peroxidases, como a reação de Trinder. Esta reação é utilizada na quantificação de componentes séricos como glicose, colesterol, triglicerídeos e ácido úrico (Martinello e Silva, 2006).
Entre os antioxidantes que têm recebido maior atenção na prevenção da oxidação lipídica e crescimento microbiano estão a vitamina $\mathrm{C}$ (ácido ascórbico) e os compostos fenólicos (bioflavonóides) (Rodrigues et al., 2003). Os bioflavonóides desempenham atividade inibitória das enzimas oxidantes cicloxigenase e lipoxigenase (Schubert et al., 1999). Além disso, destaca-se o biofla-

Tabela I. Balanço de nitrogênio e de energia e níveis de triglicerídeos e colesterol total de suínos alimentados com dietas contendo ou não extratos cítricos (EC). (Nitrogen and energy balance and triglycerides and total cholesterol levels of pigs fed diets containing or not citrus extracts (EC)).

\begin{tabular}{|c|c|c|c|c|}
\hline & Controle & EC & EPR & $p$ \\
\hline \multicolumn{5}{|c|}{ Balanço de N ( $\left(\right.$ dia $\left.^{-1}\right)$} \\
\hline Ingerido $^{1}$ & 62,79 & 63,04 & 1,79 & 0,81 \\
\hline Fecal $^{1}$ & 10,01 & 8,57 & 1,34 & 0,09 \\
\hline Urinário ${ }^{1}$ & 18,52 & 19,97 & 6,18 & 0,69 \\
\hline Absorvido $^{1}$ & 52,90 & 54,35 & 1,35 & 0,09 \\
\hline Retido $^{1}$ & 34,26 & 34,51 & 6,41 & 0,95 \\
\hline Ret/abs², \% & 99,35 & 99,37 & 0,10 & 0,71 \\
\hline CDPB, \% & 84,04 & 86,39 & 2,12 & 0,08 \\
\hline
\end{tabular}

Balanço de energia ( $\mathrm{kcal}^{-1 \mathrm{a}^{-1}}$ )

Ingerida $\quad 10443 \quad 11021 \quad 528 \quad 0,09$

$\begin{array}{lllll}\text { Fecal }^{3} & 1333 & 1214 & 130 & 0,21\end{array}$

$\begin{array}{lllll}\text { Urinária }^{3} & 212 & 229 & 71 & 0,74\end{array}$

$\begin{array}{lllll}\text { Mantença }^{3} & 2771 & 3225 & 25 & 0,19\end{array}$

$\begin{array}{lllll}\text { Retida }^{3} & 6127 & 6353 & 145 & 0,05\end{array}$

Digestível $\quad \begin{array}{lllll}3336 & 3402 & 44 & 0,03\end{array}$

Metabolizável $\quad 2962 \quad 2966 \quad 125 \quad 0,48$

$\begin{array}{lllll}\text { CDEB, \% } & 87,46 & 88,76 & 1,16 & 0,08\end{array}$

Níveis sanguíneos $\left(\mathrm{mg} \cdot \mathrm{dL}^{-1}\right)$

Triglicerídeos $\quad 172,0 \quad 221,6 \quad 56,5 \quad 0,20$

$\begin{array}{lllll}\text { Colesterol total } \quad 82,9 & 72,2 & 7,40 & 0,05\end{array}$

$E P R=$ erro padrão residual; CDPB: Coeficiente de digestibilidade da proteína bruta; CDEB: Coeficiente de digestibilidade da energia bruta.

${ }^{1}$ nitrogênio ingerido utilizado como covariável, médias ajustadas - LSMeans; ${ }^{2}$ nitrogênio retido em função do absorvido; ${ }^{3}$ energia ingerida utilizada como covariável, médias ajustadas - LSMeans. 


\section{LANFERDINI, ANDRETTA, LEHNEN, MELCHIOR, SILVA E GARCIA}

vonóide rutina, ao qual são atribuídos efeitos como elevação do colesterol-HDL e diminuição dos fatores de risco para as doenças cardiovasculares, associados à elevação na atividade da enzima antioxidante superóxido dismutase (Rodrigues et al., 2003).

\section{CONCLUSÕES}

Suínos alimentados com dietas contendo extratos cítricos apresentam um aumento na retenção de energia e no aproveitamento da energia digestível. A adição de extratos

\section{BIBLIOGRAFIA}

Ader, P.; Wessmann, A. and Wolffram, S. 2000. Bioavailability and metabolism of the flavonol quercetin in the pig. Free Radical Bio Med, 28: 1056-1067.

AOAC. 1990. Official methods of analysis of the Association of Official Analytical Chemists. Association of Official Analytical Chemists. Arlington. Virginia, DC. 1117 pp.

Cushnie, T.P.T. and Lamb, A.J. 2005. Antimicrobial activity of flavonoids. Int $J$ Antimicrob Ag, 26: 343-356.

Erlund, I. 2004. Review of the flavonoids quercetin, hesperetin, and naringenin. Dietary sources, bioactivities, bioavailability, and epidemiology. Nutr Res, 24: 851-874.

Jeauround, E.; Schumann, B. and Clunies, M.E.A. 2002. Suplementation of diets with herbal extracts enhances growth performance in newly weaned piglets. J Anim Sci, 80: 394.

Mahan, M.C.; Ching, S. and Dabrowski, K. 2004. Developmental aspects and factors influencing the synthesis and status of ascorbic acid in the pig. Annu Rev Nutr, 24: 79-103.

Martinello, F. and Silva, E.L. 2006. Mechanism of ascorbic acid interference in biochemical tests that use peroxide and peroxidase to generate cítricos em dietas para suínos reduz o nível de colesterol sanguíneo, mas não altera os níveis de triglicerídeos. A utilização de extratos cítricos na alimentação de suínos apresenta resultados variáveis e pouco conclusivos, sendo necessários novos estudos em nutrição animal.

\section{AGRADECIMENTOS}

À Comissão de Aperfeiçoamento de Pessoal de Nível Superior (Capes) e ao Programa de Apoio aos Planos de Reestruturação e Expansão das Universidades Federais (Reuni).

chromophore. Clin Chim Acta, 373: 108-116. Mellor, S. 2000. Alternatives to antibiotic. Pig Progress, 16: 18-21.

Minitab. 2007. User's guide: Meet Minitab 15. Stat College, PA. 142 pp.

Noblet, J.; Shi, X.S. and Dubois, S. 1993. Metabolic utilization of dietary energy and nutrients for maintenance energy requirements in sows: basis for a net energy system. Brit J Nutr, 70: 407-419.

NRC. 1998. Nutrient requirements of swine. NRC. National Academy of Sciences. Washington.

Padayatty, S.J.; Katz, A.; Wang, Y.; Eck, P.; Kwon, O.; Lee, J.H.; Chen, S.; Corpe, C.; Dutta, A.; Dutta, S.K. and Levine, M. 2003. Vitamin C as an antioxidant: evaluation of its role in disease prevention. J Am Coll Nutr, 22: 18-35.

Rodrigues, H.G.; Diniz, Y.S.; Faine, L.A.; Almeida, J.A.; Fernandes, A.A. e Novelli, E.L. 2003. Suplementação nutricional com antioxidantes naturais: efeito da rutina na concentração de colesterol-HDL. Rev Nutr, 16: 315-320.

Schubert, S.Y.; Lanski, E.P. and Neeman, I. 1999. Antioxidant and eicosanoid anzyme inhibition properties of pomegranate seed oil and fermented juice flavonoids. J Ethnopharmacol, 66: 11-17. 